\title{
The investigation of life-threatening child abuse and Munchausen syndrome by proxy
}

\author{
Donald Evans Centre for Philosophy and Health Care, University College, Swansea
}

\begin{abstract}
The use of covert video surveillance in the investigation of suspected life-threatening child abuse and Munchausen syndrome by proxy raises important ethical questions. That the recently reported provision of this facility in North Staffordshire was not presented to a Local Research Ethics Committee (LREC) for approval as a research exercise raises important questions about the ethical review of research and practice.

The case made for avoiding such review is first set out and then examined. The three main premisses which form the basis of the view that LREC approval is not required are identified and tested in turn. The conclusion is that there is an undeniable element of research involved in the procedure and that the welfare of all those subjected to the surveillance would be best protected by the submission of the protocol to an independent committee for ethical assessment.
\end{abstract}

\section{Introduction}

A press release by the North Staffordshire Hospital dated September 24th 1993 confirmed that the hospital had made provision for the use of covert video surveillance in cases of life-threatening child abuse. It further stated that the provision was conditional upon the establishment of a detailed and comprehensive protocol. The protocol had been prepared on an inter-agency basis with social services and the police. The release made no mention of whether the protocol had been presented to the Local Research Ethics Committee for approval. In fact no such approval had been sought. This raises the important question of whether techniques of this sort should be employed without such approval. I wish to argue that LREC approval should have been sought on this matter and that that committee has certain responsibilities with regard to the circumstances which have developed. The case highlights some important principles concerning ethical review which merit attention.

\section{Key words}

Munchausen syndrome by proxy; ethical review; covert video surveillance; good clinical practice.

\section{Local Research Ethics Committees}

The remit of Local Research Ethics Committees, according to the Department of Health guidelines (1), is to review all research involving human subjects which takes place within the National Health Service. The primary function of the committees is to protect the interests and safety of the subjects of the research. Clinical practice is not subject to ethical review in the United Kingdom, save in limited areas such as genetic engineering and the provision of assisted conception services, though there is some pressure to introduce such scrutiny.

The distinction between research and clinical practice is not always clear and this lack of clarity often figures in cases where review is avoided. It would be mistaken to underestimate the importance of evaluating the effectiveness of clinical practice: such evaluation may yield new and valuable information leading to the improvement of care. So much may be said properly to be part and parcel of good clinical practice. However, the proportion of research element in a therapeutic measure and the extent of departure from norms of practice may be significant and may constitute a threat to the interests of patients, thus calling for independent scrutiny. Whilst it would be impractical and pedantic to insist on ethical review of minor modifications of practice, the proportion of research element in a practice should be carefully controlled. We need to ask, therefore, how much the use of covert video surveillance is a departure from normal practice and how great is the research element in its use. That it is a great departure from normal practice, which embodies the consent of the competent patient, goes without saying. Whether it may be regarded as clinical practice rather than research, or clinical practice which involves research goals as merely a spin-off, is a question which I wish to pose in this paper. A negative answer will confirm the view that should already be established by the exceptional departure from normal clinical practice which the procedure entails, viz that such a procedure should be subject to the independent ethical review which has been designed for all medical research on human subjects which takes place broadly within the National Health Service. 
The most powerful case that may be put for avoiding scrutiny by a Local Research Ethics Committee may be constructed from the published accounts of the work done by David Southall at the North Staffordshire Hospital and earlier at the Royal Brompton Hospital, London (2).

The case is as follows:

i) The procedure is part of clinical practice and is not research.

ii) The child is the patient.

iii) The 'therapy' is preventive, fulfilling the doctor's duty to protect the health of the child.

\section{Each of these claims calls for examination.}

\section{I) THE RESEARCH/CLINICAL PRACTICE DIVIDE}

Dr Southall has explicitly denied that the use of covert video surveillance is part of a research procedure and has asserted rather that it is 'vital clinical work' (3), that is, good and important clinical practice. As such, of course, it would not merit consideration by a Local Research Ethics Committee. But does his claim stand up to examination? I make three objections to it:

\section{a) Good clinical practice}

If the use of covert video surveillance is clinical practice then it is purely diagnostic. No treatment is offered to the child except in the case of a violent attack being enacted upon it or a spontaneous apnoeic attack occurring in the course of the surveillance. In the latter circumstance video surveillance would in any case be redundant. If the violent attack does occur and calls for clinical intervention then it has to be faced that the opportunity for the attack is one constructed by the clinical team. Whilst it might be overstating the case to suggest that the team is cast in the role of agent provocateur the deliberate exposure of the child to the expected attack is not easily justified in terms of the interests of the child. It will not do to argue that care of the child consists in facilitating the observed assault to provide an explanation of the child's condition which can then be used to protect it from further unobserved assaults (4). Other means which do not commit the child to exposure to serious harm are available and must be preferable (5). It is a matter of concern that the team admit that parents are in fact more likely to impose airway obstruction in the contrived situation of covert video surveillance in hospital (6). They admit that '.. . parents were more likely to impose airway obstruction when, due to close nursing observation, they had been unable to demonstrate their child's "symptoms" for a period of time'. Additionally, when recordings of assaults have been made they have sometimes been assessed by the police for their adequacy for legal purposes. In cases where the evidential value was less than conclusive surveillance was continued - on more than one occasion in one case - until a satisfactory recording of a further attempt was made (7). It man. be argued that whatever else this is it is not good: clinical practice and that the protection of the child' health was not the first priority of the surveillanceg

\section{b) The concept of diagnosis}

The very idea of diagnosis in the use of covert vide $\overline{8}$ surveillance in this context is suspect. It is entirely forensic in character. Indeed to call the establish $=$ ment of a causal factor such as violent assault a forn $\vec{P}$ of diagnosis at all is to stretch the term beyond all normal medical parameters. It is vastly different, fow example, from identifying epilepsy, a patho physiological condition, as the cause. No kind of dysfunction or disease in the child is being sought: The very least that is hoped for is an elimination of the mother as a suspect - though her failure to so act during surveillance is no proof of her innocence. But this has nothing to do with medical diagnosis Neither can the idea be smuggled in successfully b 8 casting the surveillance in the guise of fulfilling the doctor's duty to protect the health of the child as show below in iii). Normally, forensic activity in medicine is separated from the activity of providing care or treatment. The forensic medical investigator is not, or at least should not be, identical with the doctor responsible for the medical care of the patient.

If the claim that the diagnosis is purely forensic i i⿱丷丅) character is countered by the claim that the procedure is designed to establish a clinical diagnosi of Munchausen syndrome by proxy then it must be noted that such a diagnosis concerns a personalit disorder in the mother, not the child. Moreover it is. a diagnosis to be made of a competent adult, at other things being equal, who has not consented to $\Phi$ clinical examination. Further problems about such diagnosis are discussed below.

\section{c) Testing a hypothesis}

There is, undeniably, a considerable element o요 research in the activity of the team employing this technique. Whilst it may have been an accident that. the first publication describing their work appearege in the clinical research section of the British Medicăb fournal (8) the content of their publications revea their research interest. In the 1987 paper the mattef is canvassed in the introduction where we are tole that covert video surveillance was used to confirfe asphyxiation by mothers as the cause of cyanotiog episodes and that 'analysis of multichannel taperecordings of physiological variables during episodes of asphyxia showed a characteristic pattern whict may be of value in alerting investigators to this cause of apnoea' (9). Matching confirmed episodes wit such patterns would enable home surveillance fơ imposed asphyxiation without video facilitie\& Though this would raise further ethical problems, I shall canvass below, it would constitute a research 
finding to show that such physiological patterns denoted violent assault. Indeed this is the promise with which the article concludes, viz: 'Future studies [sic] may show that a specific pattern of multichannel recordings of physiological variables is pathognomonic of imposed apnoea, thus avoiding the need for video surveillance'. In the 1992 paper the usefulness of such a match is again emphasised though it is still not thought to be established (6). 'Physiological recordings may help to differentiate between these different situations, particularly if data can be recorded during the episodes. This form of documentation may be invaluable not only as evidence to confirm abuse, but also to distinguish between a factitious history (no pathophysiology), a history of real symptoms (natural pathophysiology), and a history of real, but imposed symptoms (unnatural pathophysiology).' The hypothesis that such recordings could facilitate such distinctions has been challenged (10). In the 1993 paper (11) reference is again made to the fact that such physiological measurements 'may be valuable not only as an indicator of the severity of the event but also to help distinguish between fabricated events and suffocation in those patients in whom child abuse is the cause'. Thus the hypothesis is still open to refutation and the continued collection of comparative data in cases involving undisputed and conclusive evidence of abuse can be said to constitute research data against which the hypothesis may be tested.

\section{II) THE CHILD AS THE PATIENT}

The assertion that the child is the patient in the use of covert video surveillance of the circumstances in question reflects the understandable emphasis which is properly to be found in the activities of a paediatric team. However, the fact is that the role of the child as the patient is contestable and this contestability betrays the conceptual and practical uncertainties surrounding Munchausen syndrome by proxy. These questions suggest that the evolution of a series of events into a syndrome may mark the beginnings of the identity of a new clinical diagnosis such as has been seen elsewhere in the history of medicine. It is too early to tell whether this will be the result but the literature on the subject suggests that it may well be so, though there is still much disagreement about this. Some believe, for example, that the whole group of syndromes which are grouped under the umbrella term of 'personality disorders' are suspect candidates for clinical diagnosis citing, for instance, the lack of clarity of distinctions between traits and disorders of personality and the lack of any demonstrated link between such syndromes and fundamental abnormalities of an histological, biochemical or molecular kind (12). However, one does not need to take so narrow a view of clinical diagnosis to rule out Munchausen syndrome by proxy as an undisputed current diagnosis.
Munchausen syndrome simpliciter has entered DSM-III-R as a diagnostic category under the classification of Factitious Disorders (13). Though it is repeatedly said that perpetrators of the child abuse in recorded cases are not found to have a common mental illness or disease, they are said to suffer a personality disorder (14). It is generally agreed that the perpetrator of the abuse in these cases needs treatment of some sort, though the prospects of success may be slim (15). There is still considerable confusion, however, in the description of the syndrome. In one and the same paper describing the condition the child is said to have Munchausen syndrome by proxy (16), the mother is said to have the syndrome - 'the label of Munchausen syndrome by proxy may be applied to anyone who persistently fabricates symptoms on behalf of another so causing that person to be regarded as ill' (17) and the syndrome is said to be perpetrated by the mother (18). We are elsewhere told that the patient is the mother, diagnosed as having Munchausen syndrome by proxy and described as exhibiting abnormal illness behaviour (19). Significantly, perhaps, this account is that of a psychiatrist. Yet Meadow, the originator of the label, names the mother as the proxy (20) - an odd account when we are concerned with the fabrication element of the syndrome and a false one when the injury or contrived symptoms are considered.

Undeniably such behaviour patterns as are involved in Munchausen syndrome by proxy call for urgent identification and attention. Given the considerable unclarities in the very concept it cannot be claimed with any plausibility that an investigation of a whole range of cases does not involve a research element. In any case, to proceed as though the categories are clear is not good clinical practice for it is tantamount to ignoring important possible needs of one of the parties concerned in the observation. All those who have reported use of the covert-videosurveillance technique record that it has produced useful information on the psychopathology of the abusing parent. The question of consent also becomes a difficult issue where covert surveillance is used, in part, as a diagnostic technique in the care of a competent adult. Given the possibilities of considerable distress and harms that can result from mistaken suspicions in cases of alleged child abuse (5) every care must be taken to consider the welfare of both children and parents.

\section{III) PROTECTING THE PATIENT'S HEALTH}

Finally let us consider the claim that covert video surveillance is a form of protective therapy, fulfilling the duty to protect the health of the child. The response of $\mathrm{Dr}$ Southall's team to the ethical dilemma of weighing the breach of medical confidentiality against protection of the health of the child was to adopt covert surveillance to fulfil the doctor's fundamental duty to protect the health of 
the child (21). However, whilst it is a duty of medical carers to protect the health of patients they do not have a monopoly of such responsibility. Indeed, their duty, as such, is limited by the specific range of expertise and by their professional role. One does not have to belittle the role of police surveillance in order to question whether a doctor's employment of covert video surveillance solely for the purposes of detection of crime is a proper use of his skills and resources (22). He is doing it, after all, in the name of medical care and not any form of protection counts as medical care. Whilst, for example, doctors would have a duty to protect Salman Rushdie from the effects of a violent attempt on his life they do not have a responsibility to protect him from violent assault, but the police do. In cases of children at risk the social services, together with the police, have such a responsibility.

Thus, in the use of this technique we have a further distinction confused, viz that between clinical practice and criminal surveillance. Hospital authorities are not infringing the law when they install video surveillance to prevent crime on their premises. Patients and visitors have the status of guests and may be watched there as properly as in their local jeweller's store or supermarket. Privacy is not fundamentally threatened by covert surveillance. But there are other considerations at stake when it occurs in the name of medical care. Should the research aspirations of the team be realised, however, and physiological monitoring in the home become capable of identifying the behaviour of parents there, then their informed consent may well be a necessary requirement to avoid invasions of privacy which would infringe their legal rights.

\section{Conclusion}

Does the case for avoiding the scrutiny of the Local Research Ethics Committee as made out in the cited work of the North Staffordshire team stand up to examination? I think not. There is sufficient reason to believe that there are considerable research elements in the use of covert video surveillance of life-threatening child abuse and Munchausen syndrome by proxy. Certainly the interests of all patients involved should be considered. The Department of Health has issued guidance about who should decide in a given case whether a procedure should be subject to ethical review (23). It is not a matter to be determined by the practitioner. The wisdom of this advice is apparent. Not only is it important for the interests of subjects of research to be protected, they must also be seen to be protected. This serves the interests of medicine and its practitioners generally as well as the interests of patients. Furthermore where a possible tension exists between the care of a given patient and the research interests of a practitioner the value of an independent judgement is obvious.
If it comes to the notice of a Local Researcl Ethics Committee that research, or what ma possibly count as research, is proceeding in its area: without proper consultation with the committees? then it has the responsibility to report the same to the district health authority, the relevant NHS body and the relevant professional bodies (24).

Of course this is not to anticipate whether or nog the local committee will advise the district healtho authority that it approves or refuses to approve of the practice. It is to guarantee that the activities oP researchers are conceived with care for the interests and welfare of all research subjects and that they ares seen to be so conceived.

Donald Evans, BA, PhD, is Director of the Centre for Philosophy and Health Care at the University of Wales $\overrightarrow{20}$ Swansea.

\section{References and notes}

(1) Guidelines: Local Research Ethics Committees. London Department of Health, 1991.

(2) Southall D P et al. Apnoeic episodes induced by smothering: two cases identified by covert videœ surveillance. British medical journal 1987; 294? 1637-1641. Shinebourne E A, Southall D P. Chilp abuse: observation, analysis, action. IME bulletin 1988 Sept: 13-14. Samuels M P, Southall D P Munchausen syndrome by proxy. British journal oळ hospital medicine 1992; 47, 10: 759-762. Southall D I et al. Fourteen cases of imposed upper airway obstruction. Archives of diseases in childhood 1992;67. 162-170. Southall D P et al. Diagnosis and manage ment after life threatening events in infants and young children who received cardiopulmonary resuscitation? British medical journal 1993; 306: 489-492.

(3) Southall D P et al. Video traps catch abuse of childrer by parents. The Observer 1993 Sept 26: 9.

(4) See reference (2): British medical journal 1993: 614

(5) Foreman D M, Farsides C. Ethical use of cover videoing techniques in detecting Munchauserm syndrome by proxy. British medical journal 1993;307; 611-613.

(6) See reference (2): Archives of diseases in childhood: 168

(7) See reference (2): Archives of diseases in childhood: 165…

(8) See reference (2): British medical journal 1987: 294ก.

(9) See reference (2): British medical journal: 1637.

(10) See, for example the letter of Morley C J. Experts differ over diagnostic criteria for Munchausend syndrome by proxy. British journal of hospital medicing 1992; 48: 197, and a further letter of Morley et at Fourteen cases of imposed upper airway obstruction Archives of diseases in childhood 1992; 67: 1519.

(11) See reference (2): British medical journal 1993: 306.

(12) See, for example, Chatterjee A, Tosyali M Ci 'Munchausen's-by-proxy'. Lancet 1994; 343: 1040울

(13) See: Diagnostic and statistical manual of mental disorder [3rd ed], revised. Washington: American Psychiatrig Association, 1987: 301.51, 300.16 and 300.19.

(14) Meadow R. Management of Munchausen syndrome by proxy. Archives of diseases in childhood 1985; 600 392. Meadow R. Suffocation, recurrent apnoea, an\& sudden infant death. Paediatrics 1990; 117, 3: 35 
Meadow R. Munchausen syndrome by proxy. British medical journal 1989; 299: 250. Reference (2): Archives of diseases in childhood: 169.

(15) See, in addition to already cited papers, Nichol A R, Eccles M. Psychotherapy for Munchausen syndrome by proxy. Archives of diseases in childhood 1985; 60: 344-348.

(16) See reference (14): Archives of diseases in childhood: 389.

(17) See reference (14): Archives of diseases in childhood: 385.

(18) See reference (14): Archives of diseases in childhood: 393.
(19) See reference (15): 348 and 346.

(20) See reference (14): British medical journal: 248.

(21) See reference (2): British medical journal 1987: 1640.

(22) Southall D P. Ethical use of covert videoing for potentially life threatening child abuse: a response to Drs Foreman and Farsides. British medical journal 1993; 307: 614 .

(23) See a letter of guidance from the Department of Health to the Association of Community Health Councils for England and Wales, and another to Royal Liverpool Children's Hospital from the Department of Health, each dated 13 May 1992.

(24) See reference (1): 3.22.

\section{News and notes}

\section{New editor}

On the 1st September 1994 Dr Charles Hind took over from Dr Barry Hoffbrand as editor of the Postgraduate Medical fournal. Dr Hind is a Consultant Physician in General and Respiratory Medicine at the Royal Liverpool University Hospital and the Cardiothoracic Centre, Liverpool. The first issue to be published under the new editor appeared in January 1995, in a new A4 format, and contained a mixture of original articles, short reports, editorials and review articles.

The new editor hopes that the journal will help doctors in training to acquire the necessary skills to enable them to deliver the highest possible standards of patient care. There will also be articles designed to help those doctors' trainers to develop suitable training programmes for their trainees. And finally, once that training is complete, the journal will allow doctors to maintain those high standards by a process of continuing medical education.

For further details, please contact: Mrs J M Coops, Postgraduate Medical fournal, 12 Chandos Street, London W1M 9DE. 